\title{
Polysémie des mots injurieux et affectueux en français et en arabe égyptien
}

\author{
DR. Taha Roshdy Taha \\ Professeur adjoint en Linguistique française \\ Université de Kafrelsheikh
}

Très peu d'études ont été explicitement consacrées aux termes d'injure $^{1}$ et d'affection en français et en arabe égyptien. Les termes d'injure et ceux d'affection appartiennent à ce qu'on appelle la sociolinguistique, cette discipline qui traite la manière avec laquelle n'importe quelle société se sert des idiomes pour remplir son vouloir dire. Etant donné que les termes en question s'emploient pour exprimer des sentiments humains: mécontentement, colère, tendresse et amour, ils sont par conséquent, semblables et parfois identiques dans presque toutes les langues sans oublier pour autant les spécificités idiomatiques de chacune des langues. Les diverses interactions verbales entre les deux langues: l'arabe égyptien et le français, via les rapports à la fois conflictuels et amicaux, firent en sorte que les injures et les termes affectueux prennent leur chemin de la première langue à la deuxième et vice versa. Cela se montre clairement lorsqu'on procède à l'étude des termes

1-Bubois et étal, definissent l'injure en disant qu' « on appellee injure toute parole, toute attiude ou allusion à contenu symbolique perçue et vécue par le sujet injurié comme déalorisante et blessante pour lui.»> concernés dans l'arabe égyptien et leur équivalent en français.

La problématique de ce travail consiste à élucider les points de convergence et ceux de divergence dans les deux langues. Nous essayerons également de répondre à la question primordiale suivante: quelle est la valeur sémantique et pragmatique de l'emploi de tels termes et leur impact sur les destinataires?

Nous allons étudier également les deux termes en français et en arabe égyptien pour savoir leur influence sur les personnes. Nous commencerons par les expressions injurieuses qui portent en arabe égyptien, comme c'est le cas dans le français, des connotations négatives sur les allocutaires. Elles représentent un grand nombre d'informations concernant le destinateur lui-même et fonctionnent à ce niveau comme des marqueurs sociaux. Leur rôle linguistique est généralement lié à des variabilités sociales concernant la personne qui parle tels que l'âge, le sexe et plus précisément le milieu social.

Comme nous le verrons dans notre étude, les expressions d'injure sont classées en deux langues en fonction du registre de langue dont ils relèvent. Nous pouvons dire que la 
corrélation entre le choix de langue et les classes sociales différentes dans la société reflète le milieu social du destinateur. Si le niveau linguistique des termes employés descend vers le bas, c'est-à-dire vers la langue vulgaire, nous descendrons au niveau inférieur de la stratification sociale. Nous examinerons l'emploi de ces termes en tant que marqueurs sociaux dans les différents milieux sociaux dans les deux langues. Nous allons passer en revue les expressions injurieuses les plus fréquentes en nous basant sur les champs lexicaux les plus courants dans les deux langues.

\section{Termes d'injures dans l'interaction quotidienne}

Les injures sont très courantes dans la langue parlée entre les différentes personnes, c'est-à-dire qu'elles sont très fréquentes dans les interactions quotidiennes. Elles sont très employées dans les moments de vraie colère où les personnes expriment leur agacement, leur dégout ou même leur indifférence les uns avec les autres. D'autre part, nous avons des termes injurieux qui sont utilisés entre les amoureux à des fins humoristiques. Dans les milieux égyptiens et ceux de français, ces termes ne représentent pas une menace pour la face du destinataire, ni pour celui qui les profère.

Les expressions injurieuses utilisées dans leur vrai sens sont toujours précédés de la particule vocative [ya] qui donnent l'équivalent français de " espèce de...». En d'autres termes, ces mots sont classés suivant leur place de vulgarité appelée par Parkinson «the heaviness scale». Voici quelques termes:

-Ya homar, l'équivalent en français est: Espèce d'âne.

-Ya Kalb, l'équivalent en français est: Espèce de chien.

-Ya Khannzeir, l'équivalent en français est: Espèce de cochon.

Chaque injure est fortement liée à la classe sociale de celui qui tient la responsabilité de la parole. Rappelons que les personnes de la classe populaire ne trouvent aucune pudeur à utiliser les expressions interdites en parlant avec les membres de la même classe. Contrairement à ce milieu inferieur, les classes supérieures ou moyennes refusent complètement de proférer ces termes blessants. Le choix de ces termes est lié de premier ordre au degré de colère du locuteur et son interaction au moment de l'énonciation. En règle générale dans les deux langues de notre corpus, plus nous sommes en colère ou un moment de rage, plus nous descendons au niveau inférieur de l'échelle des expressions injurieuses. Les personnes qui représentent la classe moyenne peuvent utiliser ces termes lourds lorsqu'elles sont en colère ou excitées dans une situation difficile. Notons également que le niveau personnel de politesse joue un rôle très important dans l'usage de ces expressions jugées tabous dans les normes sociales de chaque peuple. Une minorité de la classe moyenne utilise ces expressions blessantes, tandis que la majorité française et égyptienne appartenant à cette classe refuse catégoriquement d'employer ces injures quel que soit 
leur niveau de vulgarité et n'utilise que quelques mots dits «légers») dans le cas de l'extrême colère. Par contre, pour beaucoup de personnes qui appartiennent à la classe populaire, l'emploi de ces injures est une partie essentielle de leurs habitudes quotidiennes et ils les utilisent avec leurs proches même avec les petits!

Mais l'emploi des injures diffère dans les deux sociétés. Les jeunes français emploient ces termes devant les âgés et sans avoir honte de les articuler même en présence de leur père ou de leur mère, le mot «putain!» par exemple peut être utilisé à la maison ou à la réponse d'une question:

Le père à son fils qui vient en retard à la maison: Ce n'est pas un hôtel ça! Je t'avertis la deuxième fois, je vais te mettre à la porte!!!

Le fils répond: Ouf! Putain!

En arabe égyptien, nous pouvons rencontrer la même chose dans le même contexte mais ce n'est pas le même niveau. Il y a quand même un peu de respect et la politesse reste encore vivante malgré le grand changement langagier chez les jeunes et le bouleversement des liens familiaux. Ceci s'explique par la place accordée habituellement aux vieux qui implique non seulement l'interdiction d'utiliser ces mots avec des destinataires âgés, mais aussi de proférer des termes injurieux en présence de ces personnes comme le père par exemple. Ces termes injurieux comme « Haga Teqrif!», c'est-à-dire en français «c'est dégoutant! Ou c'est galère!»» n'est pas utilisés en présence de nos pères ou grands-pères même les voisins. Dans les milieux égyptiens, le sexe joue une place très importante dans cette problématique et le choix de ces termes sont bien filtré, surtout chez les femmes avant de parler. Il est vrai que les sexes émettent et reçoivent les mêmes injures de façon égale mais la différence entre les deux se situe au niveau de la possibilité d'emploi dans des situations en public. Les hommes sont courageux en disant un terme injurieux dans des situations publiques, notamment quand ils sont en colère, alors que les femmes, par pudeur, n'en ont pas le droit. Les coutumes et les mœurs interdisent les femmes à pratiquer les mêmes choses ainsi que les sentences religieuses.

Rappelons que ces expressions peuvent être utilisées entre les personnes inconnues dans les situations variées de la vie quotidienne en cas de colère aussi bien entre les amis et les camarades pour plaisanter ou pour exprimer leur réaction contre ce qui blesse les sentiments. Voici un exemple: es âne!

-Enta Homar!, c'est-à-dire: Tu

Nous pouvons dire que ce terme est utilisé entre les intimes pour rigoler, mais d'une manière sérieuse, il est employé dans les différentes situations de la vie pour exprimer les réactions négatives en face de quelqu'un ou quelque chose qui causent directement des problèmes. Ces termes accroissent dans les milieux populaires pauvres. 


\section{Classification lexicale des expressions injurieuses}

Malgré la grande différence entre les termes injurieux en arabe égyptien et en français, les deux langues ont beaucoup de termes dans ce domaine. Les français publient des livres et des dictionnaires qui expliquent et analysent tous les mots d'injure qui relèvent des niveaux dits: légers, moyens, lourds. Les égyptiens ont aussi un amas des injures et des insultes qui dépasse dix mille d'après les dernières statistiques. Pour les deux langues, les champs lexicaux sont vastes et embrassent plusieurs sortes d'injures. Nous commencerons par les noms des parents qui s'emploient beaucoup dans toutes les classes égyptiennes. Voici quelques exemples:

-Yenhal Omak! C'est-à-dire en français, Que Dieu maudisse ta mère!

-Yenhal Abouk! C'est-à-dire, Que Dieu maudisse ton père!

-Abouk Marabaksh!, C'est-à-dire, tu es mal éduqué par tes parents!--- uniquement pour les garçons.

-Abouki Marabakishi!, C'est-àdire, tu es mal éduquée par tes parents!--- uniquement pour les filles.

Nous avons beaucoup de termes injurieux concernant les parents dans tous les domaines de la vie, les interlocuteurs utilisent ces expressions avec intention formelle et délibérée pour humilier et énerver les autres personnes. Les injures par mère ou par père causent un traumatisme et une blessure pour les destinataires. En français, il y a beaucoup d'injures mais ne se situent pas au même niveau comme dans les cas égyptiens. Voici quelques échantillons:

-Fils de pute!

-Bâtard!

-C'est un maquereau!

Les trois termes injurieux sont beaucoup employés dans les quartiers où habitent les étrangers. Le dernier terme possède beaucoup d'équivalents, il est l'équivalent de «proxénète», c'est-à-dire la personne qui tire profit de la prostitution d'autrui ou bien la favorise. Nous pouvons dire également, Enfant de maquereau! Ou Enfant de putain! Et Enfant de pute!

La série de ces injures ne cesse de se réactualiser en fonction du temps. Voici également quelques exemples:

Ta gueule!, enculé, va te faire foutre, sale con, salaud, salope, connard, femme laide, gros dégueulasse, merde.

Le deuxième champ lexical dont relève une grande quantité des expressions injurieuses est pratiquement celui des noms d'animaux soit sauvages ou domestiques. Cette catégorie d'injure est différente d'un pays à l'autre, il y a des peuples qui acceptent d'une manière satisfaisante les appellations des noms d'animaux et par contre, il y en a qui refusent totalement cette appellation qui sont pour eux des insultes directes condamnées par la loi. 


\section{Les termes courants d'injure}

Notons que le choix de ces termes dans les deux langues est bien sélectionné et son objectif est d'agacer et déranger les destinataires. Chaque nom d'animale contient quelque chose de péjoratif vis-à-vis du destinataire et sa manière de parler ou de raisonner. Le but essentiel en prononçant ces gros mots est de montrer les défauts des personnes concernées. Le mot clé ou générique de ces champs lexicaux est (Hayawan/hayawana) en arabe égyptien, la même chose et la même influence en français viennent de mot (bête) pour le masculin et le féminin. Nous pouvons avec beaucoup de facilité trouver ces deux termes dans la vie quotidienne en France et en Egypte. Voici quelques échantillons:

-Enta Hayawan! Ou Enty Hayawana! En français: Tu es bête!

Nous pouvons dire en français: C'est une question bête! C'est-à-dire dépourvue d'intelligence. Un homme bête! ${ }^{2}$ C'est-à-dire un homme stupide. $\mathrm{Ce}$ mot, en français, est toujours adjectif mais il porte le synonyme d'un nom. Nous pouvons le remplacer par 〈(sot»», « «stupide». En arabe égyptien, son sémantisme est le même qu'en français mais nous ajoutons qu'il signifie: il ne comprend rien ou il est ignorant, c'est-à-dire qu'il n'a aucun savoir. Dans les deux langues, le destinataire ne se comporte pas comme un être humain mais comme un animal. Les animaux qui

1-www.academia.edu s'emploient beaucoup dans ce domaine en arabe égyptien sont (Homar/Homara) Homar pour le masculin et Homara pour le féminin. En français, le mot (âne m. /f. ou ânesse) est un terme qui montre également la stupidité flagrante du destinataire. Voilà des exemples:

-Enta Homar Mabtifhmsh! (Tu es âne! Tu ne comprends pas!)

-El Homara ely Bititkalem! (L'âne qui parle!) Un professeur en s'adressant à une étudiante qui fait du bruit ou bavarde dans la classe. Dans l'arabe égyptien, l'âne joue un rôle essentiel quand nous voulons humilier quelqu'un et l'obliger à respecter la loi ou les règles suivies dans la société. En français moderne comme l'ancien français, l'âne est également le symbole de l'ignorance et la stupidité. Il est polysémique et son rôle est varié. L'expression la plus courante dans les milieux populaires français est (être comme l'âne), c'est-à-dire qu'il est idiot et ignorant comme l'âne. Il est l'équivalent des mots suivants: bourrique, bête, baudet, mule, mulet. Nous pouvons entendre facilement ces termes en lançant des injures.

-Il est mule!

-Tu es bourrique, toi! C'est-àdire une personne sotte et entêtée.

Ce terme est aussi employé pour manifester un mépris et une colère à un imbécile. Dans ce cas, l'âne est le synonyme des adjectifs suivants: sot, imbécile, ignorant, buse, crétin, stupide, etc.

$$
\begin{aligned}
& \text { Il est âne. ----- Il est sot. } \\
& \text { Il est âne. -----Il est imbécile. }
\end{aligned}
$$


Il est âne. ------Il est ignorant.

Les proverbes et les expressions français utilisent le mot (âne) pour les mêmes finalités, c'est-à-dire qu'on qualifie quelqu'un d'âne pour designer qu'il est hébété et n'arrive à bien raisonner. Voici quelques proverbes montrant les mêmes connotations:

-L'âne frotte l'âne. C'est-à-dire que les imbéciles s'encouragent entre eux.

-âne bâté. Qui ignore beaucoup de choses par stupidité.

-Oreilles d'âne. Les longues oreilles symbolisent la pesanteur d'esprit.

En arabe égyptien, le petit de l'âne est nommé (Gahsh), c'est-à-dire (ânon) en français, ce terme est fréquemment utilisé pour montrer le caractère têtu et peu intelligence de la personne. Il est beaucoup plus blessant que le mot (Homar) et son équivalent dans la vie quotidienne égyptienne est (Teiss), (bouc). Les professeurs qualifient les étudiants qui ne comprennent pas leur leçon et restent la bouche bée par le mot (Teiss). Voici un exemple:

-El walad da Teiss Kebeir! C'est-à-dire qu'il est un grand bouc.

Pour le reste des noms d'animaux, les lexiques égyptiens des injures sont plus riches que le registre français de cette catégorie. Nous pouvons citer quelques injures courantes dans la rue égyptienne.

-Ibn elKalb! (le fils du chien)

-Bent elkalb! (la fille du chien)

Ces deux injures marquent la bassesse et la vilenie. Les mots (Kalb et kalba) qui impliquent les mêmes connotations sont habituels en Egypte surtout entre les jeunes garçons et les jeunes filles mais la loi punit les interlocuteurs qui emploient ce gros mot dans les places publiques. Par plaisanterie, on peut utiliser ces termes entre les amis et les proches. Voici un exemple:

-El Kalb da mosh byzakr drossoh! Un père qui adresse la parole à son fils qui n'étudie pas ses leçons. Il dit: «ce chien n'étudie pas ses leçons!»>

-Elkalba di wahshani! Une jeune fille dit à son amie en plaisantant: «cette chienne me manque beaucoup!»>

Contrairement à l'arabe égyptien, le terme «chien» en français n'est pas une insulte mais il est le symbole de la fidélité et l'amitié. $\mathrm{Si}$ on dit à quelqu'un: $\mathbf{T u}$ es fidèle comme le chien, il va répondre en disant: Merci monsieur, c'est la gentillesse de votre part! Il représente donc des qualités comme l'honnêteté et la loyauté.

Nous avons un autre terme d'injure animale qui joue un rôle important dans les deux langues, c'est le mot «khanzeir») en arabe égyptien et «cochon») en français. Dans la société française, le cochon est un gros mot et la loi punit les personnes qui l'utilisent. Si on dit: sale cochon! Le destinataire qui reçoit ce terme réagit vite et d'une manière agressive contre l'interlocuteur. La loi française interdit son usage dans la presse écrite et électronique ainsi que dans la rue et les lieux publics. Le chanteur belge 
Jacques Brel a insulté les bourgeois en utilisant le terme 〈(cochon») dans sa chanson, il dit: " Les bourgeois, c'est comme les cochons, plus ça devient vieux, plus ça devient bête!) ${ }^{3}$

Nous avons également vu, par hasard, en lisant le journal électronique «Midi-Libre» un commentaire d'une lectrice française Christine Tasin le 19 février 2013, elle a dit: <et si à la place du cheval, ça avait été du cochon? "). Son commentaire est resté un quart d'heure sur le site avant d'être censuré. Elle a montré que les responsables du journal électronique ont répondu:

«Bonjour, certains de vos commentaires sont contraires à notre charte qui proscrit notamment les propos injurieux, racistes, diffamatoires, agressifs.»>

Le mot cochon marque aussi en français la saleté et l'indifférence totale. En arabe égyptien, nous avons la même fonction et la même influence.

Les défauts physiques sont aussi importants dans ce domaine et leur champ lexical est riche et polyvalent. Le dictionnaire quotidien de l'arabe égyptien contient beaucoup d'échantillons. Nous avons des termes qui blessent les destinataires surtout les handicapés comme «Ahrg»" (boiteux m. boiteuse f.), «Aqrh»» (chauve), «Ahma» (aveugle m. /f.), ce dernier terme est généralement adressé aux personnes inconnues qu'on heurte dans les rues sans qu'ils présentent leur pardon ou à tous ceux qui traversent la rue sans faire attention aux véhicules. Voici un exemple:

-Hassib ya Ahma! Enta mabitshofsh!, c'est une phrase très courante dans la rue égyptienne, les chauffeurs du taxi répètent ces termes pour avertir les passagers qui sont distraits en passant la rue, (Attention aveugle! Tu ne vois pas!). En français, ce terme existe et il est considéré comme injure. Sur le site www.20minutes.fr, nous avons lu un article intitulé: Cédric Mourier, arbitre: «Un joueur qui dit "tu es aveugle", il faut le comprendre ${ }^{4}$. Nous avons constaté que celui qui a fait cette rencontre avec l'arbitre qualifie le terme (aveugle) comme normal malgré sa connotation injurieuse mais il avertit les joueurs de proférer les insultes parce que les supporters regardent le match à la télévision. Nous allons citer deux questions et leur réponse:

Entendez-vous souvent des insultes?

Oui bien sûr. D'abord, il faut comprendre la langue. Beaucoup disent des choses horribles dans une langue qu'on ne comprend pas. Il y a les insultes de base, les classiques. Il y a les insultes à soi-même. Si on est sur un court couvert par la télé et que tout le monde entend l'insulte. Vous êtes moins flexible que si c'est sur une courte annexe où on peut considérer ça comme une discussion privée

${ }^{4}$ Publié le 29/01/2014 
d'hommes. Entre nous. Ici, il y a des micros tous les mètres. Plus il y a la télévision plus les joueurs font attention à leur image. Si un joueur dit un 〈〈putain») dans les bâches et qu'à la télévision on n'entend que ça, le téléspectateur ne comprendrait pas forcement que le joueur dise cela et que l'arbitre reste impassible.

\section{Vous faites donc semblant de ne pas entendre parfois...}

Ce n'est pas ça, mais on ne doit pas franchir la limite. Le «putain» ou le « fuck» crié fort après une annonce dépassent la limite. Maintenant un joueur qui dit « tu es aveugle», il faut le comprendre. Ce n'est pas irrespectueux.

Nous avons constaté que la réponse de l'arbitre est complètement logique et acceptable mais les injures et les insultes restent des gros mots malgré leurs diverses connotations. Le registre égyptien a d'autre termes qui donnent un sens pareil, c'est le mot (Atrash/ Tarsha) (sourd/sourde) qui sont également employés pour s'adresser aux passants par exemple à qui les chauffeurs doivent klaxonner pour qu'ils fassent attention. Dans ce contexte, nous signalons que les deux termes (aveugle/sourd) ne s'emploient jamais au sens propre du terme c'està-dire que ces expressions ne concernent les vrais aveugles ou les vrais sourds. En français, le terme possède la même fonction. On peut entendre « $\mathrm{Tu}$ es sourd!» dans la rue surtout dans les quartiers populaires où habitent les étrangers. Dans les deux langues (française/égyptienne), la loi punit également les personnes qui profèrent ces mots.

Ces termes d'insulte montrent

les imperfections morales des destinataires, c'est-à-dire les défauts qui caractérisent ces derniers. Les injures qui figurent le plus fréquemment dans la société égyptienne sont: (qalil el adab/qalilt el adab) (impoli pour le masculin, impolie pour le féminin), elles sont adressées aux personnes qui énoncent des insultes blessantes ou font un geste interdit par la main. On peut dire: (El walad da qalil el adab wi mtrabash!) (Littéralement: Ce garçon est impoli ou manque de politesse et il est mal éduqué!). En français, ce terme porte la même connotation. Tous ces termes sont largement utilisés et ils font référence au même champ lexical.

Les défauts mentaux et psychologiques comme l'ignorance, la folie, la stupidité, l'idiotie jouent aussi un rôle important dans les deux langues surtout l'arabe égyptien. Les mots les plus courants que l'on entend le plus souvent dans tous les milieux égyptiens sont: (Ghabi/Ghabia) (stupide $\quad \mathrm{m} / \mathrm{f}) ; \quad$ (Ahbal/Habla) (fou/folle). On peut entendre cette phrase dans les deux langues et son sémantisme est pareil: Tu es fou! Adresser à quelqu'un qui agit comme les fous. Les deux expressions sont donc identiques en français et en arabe égyptien.

Citons enfin quelques termes qui possèdent des sens injurieux et qui sont fortement utilisés en Egypte. Tel est le cas de l'expression (lama yegy 
ebn el kalb habaho!) (Littéralement Fils du chien! Quand il viendra, je l'abattrai!) qui a trois connotations: la menace, l'injure et la malédiction. Les parentes utilisent beaucoup cette expression pour montrer leur colère contre les enfants qui sont en train de commettre une faute quelconque, pour les corriger. Mais pour les personnes égales lorsque quelqu'un fait tort à quelqu'un d'autre, l'agressé invoque la malédiction à l'agresseur en disant cette expression.

Quant aux termes péjoratifs et vulgaires, ils représentent une grande partie des vocabulaires en français et en arabe égyptien. Ils possèdent des connotations sexuelles qui s'énoncent d'une manière directe ou métaphorique.

Les termes d'affection possèdent la même place et la même importance dans cette étude, pour cela nous allons aborder et analyser quelques termes dans les deux langues dans leur fonction communicative.

\section{Variété des mots d'affection}

Le champ lexical des termes d'affection est également plus vaste, plus riche et plus utilisé en arabe égyptien et en français courant pour s'adresser aux proches, aux amis, aux membres de la famille, aux amoureux et même aux enfants. Les mots d'affection sont omniprésents dans toutes les interactions verbales de chaque peuple et chaque locuteur exprime de sa manière les termes assortis qu'il choisit pour attirer l'attention de son partenaire ou manipuler les autres. Les auteurs du Dictionnaire de linguistique et des sciences du langage (1994:20) donnent un sens au mot affectif en disant $\mathrm{qu}^{\prime}<<$ on appelle langage affectif ou expressif celui qui traduit l'intérêt personnel que nous prenons à nos paroles par une manifestation naturelle et spontanée des formes subjectives de la pensée. $>>$ Nous allons passer en revue les termes essentiels qui remplissent les fonctions pragmatiques de cette catégorie sociolinguistique.

Le nombre des termes d'affection en arabe égyptien est très grand et laisse la liberté à chaque individu de créer des mots qui se construisent immédiatement pendant l'opération communicationnelle et en fonction de l'état moral de l'interlocuteur et la satisfaction complète de l'allocutaire en recevant la parole. Le choix des mots, dans ce cas, est donc réservé à la créativité individuelle. Le registre de cette sorte des mots contient beaucoup d'exemple et beaucoup de situations vivantes.

Nous commençons par les termes diminutifs qui occupent une grande place dans la société égyptienne car les pères et les mères et même tous les membres de la famille sont les premiers à les utiliser avec les petits enfants. Voici quelques exemples:

-Enta Fin Ya Hamada, Une phrase adressée par une mère ou un père à leur petit qui s'appelle (Ahmed ou Mohammed), (Hamada, tu es où?).

-Zanoba Habibt Baba!, diminutif adressé par papa à sa fille 
Zeinab, (Zanoba, l'amour de papa!)

Dans les deux exemples précédents, les termes (Hamada/Zanoba) sont employés avec affection par les deux parents qui s'adressent à leur fille en créant par eux-mêmes un diminutif à partir du prénom de cette dernière. La plupart des mots diminutifs en arabe égyptien terminent par le pronom possessif de la première personne du singulier (I). L'emploi de ce pronom à la fin d'un nom donne au diminutif un aspect plus personnalisé et plus émotionnel. Le père peut facilement dire à sa petite fille qui s'appelle Warda (Warditi) (Ma fleur) et (Salomti) pour le nom de Salma.

Le diminutif ne concerne pas uniquement le père, la mère et les membres de la famille, mais concerne également les amoureux, les amis, les collègues, les couples mariés qui ne l'utilisent que dans des moments très intimes. Les situations entre intimes comportent une catégorie de termes dont les sens font directement référence aux sentiments de l'émetteur. Les premiers termes qui expriment l'amour par excellence est (Habibi/habibty) (littéralement, l'amour pour le masculin et le féminin) qui peuvent aussi posséder une forme diminutive notamment en parlant aux enfants comme (Habubit papa/Habubi). Les petites filles sont les premières qui reçoivent ces mots et les mères les utilisent fréquemment comme (Habib mama/Habibet mama) (l'amour de maman $m$. f.). Les pères n'utilisent ces termes que dans quelques cas individuels. Le terme égyptien (heta men Qalbi) est une expression commune entre les petits enfants et les amoureux. On peut entendre un fiancé dit à sa fiancée: (Enti qabi) (Tu es mon cœur) et la même phrase utilisée pour s'adresser aux enfants: (Enti qalbi ou Roh mama) (le coeur de maman). Le mot (Roh) (Mon âme) est également une expression qui s'emploie toujours entre locuteurs comme les amoureux. Voici quelques exemples: (Enta Rohi! Enti Rohi!) (Tu es mon âme!). Les termes (Omri/Hayati/Donyeti) (littéralement, ma vie) sont les semblables de mot (Habibi).

En français, les termes diminutifs existent partout dans les différents milieux sociaux. Mais avant de citer quelques exemples, nous pouvons montrer la définition de ce mot. Les diminutifs «sont fréquemment utilisés comme hypocoristiques, c'est-à-dire pour ajouter une nuance affective, caressante au mot considéré. Ils sont ainsi fréquemment formés sur les noms propres où ils correspondent à une forme de surnom. $)^{5}$

Cette catégorie linguistique est donc un acte de dérivation lexicale ajoutant aux mots l'idée de petitesse afin qu'ils soient capables d'exprimer les sentiments intérieurs des personnes dans les situations affectives. Jean Dubois et les autres dans le Dictionnaire de linguistique

5

www.dictionnaire.sensagent.leparisien.fr/ dimunitif/fr-fr/ 
et des sciences du langage, (1994:148) définissent cette catégorie linguistique en disant que "LLe diminutif est un nom qui se réfère à un objet considéré comme petit et en général accompagné d'une connotation affective (hypocoristique). Ce sont les conditions d'emploi (contexte affectif ou familier) qui caractérisent le diminutif. Le diminutif peut être une forme réduite (Steph pour Stéphane, Ed pour Eduard), une forme réduite redoublée (Nini pour Véronique) ou le nom redoublé (Jean-Jean) ou une forme suffixée (Jeannot pour Jean) 》).

Le registre français est riche de ces vocabulaires. On peut entendre un père adressant à son petit-fils en disant: «Pierrot, viens ici!» ou « Mon petit Jeannot est beau!». Les français utilisent également le terme "« Mon amour» en parlant avec les petits et les grands. Mais parfois, on rencontre quelques termes étranges fréquents entre les jeunes comme «Ma puce!» qui sont utilisés pour exprimer l'amour ou un geste d'affection. Ces mots sont considérés comme des expressions de l'intimité.

Il faut rappeler à ce propos que les termes d'affection dans la langue française remontent à la constitution même de la langue après sa sortie du latin. Cela était indissociable de la vie mondaine qui atteint son épanouissement dans les salons, surtout littéraires au temps du classicisme « le XVIIème siècle» et $\mathrm{au}$ temps des Lumières « le XVIIIème siècle»». La raffinité du langage de ces lieux respectueux où se trouvaient les dames de la haute classe « comtesses, marquises, duchesses .etc. $\rangle$ fit en sorte que les termes affectueux restaient dans le cadre du décent est subsiste très loin de blesser les sentiments et le goût public. En Egypte, vu la vie conservatrice suivie par une phase d'ouverture incontrôlée, les termes d'affection ont subi parfois des allusions indécentes, voire érotiques.

Il existe une autre catégorie de ces termes qui servent à encourager les jeunes et les petits enfants à faire quelque chose de bien ou à les complimenter après une réussite dans les différents domaines de la vie. On trouve des mots tels que «Bravo!», «impeccable!», «parfait!», «(courage!» qui poussent les allocutaires de façon à ce qu'il se sente «bien dans sa peau». En arabe égyptien, on emprunte le mot français «Bravo!» pour les mêmes fins. On trouve également des termes comme «Bravo alik» dans toutes les situations de la vie. Si un étudiant a de bonnes notes dans son bulletin scolaire, les parents lui disent: «Shater Bravo Alik!» «Habile, Bravo!», mais si le petit enfant fait son devoir chaque jour et étudie bien ses petites leçons, on lui dit«〈Shator», l'emploi du diminutif ici est une forme hypocoristique qui exprime une intention tendre et affectueuse.

Notons aussi que les connotations de certains de ces termes d'affection contiennent des mots qui appartiennent aux champs sémantiques de la beauté comme «Enta zay el amar!»»« littéralement, 
Tu es luisant comme la lune!» qui prend aussi une forme de diminutif très courante en Egypte comme «Amur/Amura». Nous avons les mêmes résultats si nous utilisons les termes suivants: «Hilw/Hilwa» «Joli/Jolie», «Assal» «Miel», «Sukar/Sukara» «Sucre, pour les deux sexes». Ce dernier terme est courant dans toutes les classes en Egypte, on l'utilise pour s'adresser aux personnes dont la gentillesse est grande, mais aussi pour les personnes qui sont comiques et mignonnes. L'exemple le plus célèbre est «KKalamo zay el sukar!» «Ses paroles sont douces comme le sucre!».

Rappelons que l'usage de ces termes d'affection dépend de la personnalité de l'interlocuteur et son choix des mots et à qui il adresse sa parole. La nature de son acte de parole est parfois une sorte de flatterie, de courtoisie ou de politesse. Parkinson $(1985: 190)$ explique en anglais cette idée en disant que:

«the same term would be head as mugamla (courtoisie, politesse) addressed one's child, girl friend or spouse, would certainly be head as mu@aakasa (taquinerie) to a stange girl or even to a neighbor girl, a female teacher or any female with whom speaker is expected to have a relationship marked by formality or respect>>

L'arabe égyptien et le français sont donc deux langues qui comportent beaucoup de termes d'affection et leur objectif est d'encourager le destinataire de façon à le rendre gai de ses compétences, de ses performances ou de ses capacités. Les termes d'affection jouent un rôle très important dans la communication parce qu'ils permettent aux locuteurs d'inventer leurs termes et d'exprimer librement leurs sentiments à leurs partenaires. Pragmatiquement, les termes d'affection laissent la porte ouverte devant les néologismes qui envahissent les deux langues surtout l'arabe égyptien à travers le nombre de mots que les interlocuteurs empruntent à d'autres situations qu'ils sont vécues ou vues au cinéma et à la télévision. Le portable est maintenant un moyen efficace aux allocutaires de connaitre une ou plusieurs langues étrangères et comprendre l'usage de nouveaux termes dans ce domaine.

Pour terminer cette partie, nous pouvons dire que chaque peuple possède son répertoire de termes d'affection que seuls les natifs comprennent par cœur leur emploi et leur signification.

En guise de conclusion, nous pouvons dire que les expressions d'injure et celles d'affection constituent un très vaste registre dans toutes les langues dont le français et l'arabe. Cela fait partie intégrante de la sociolinguistique qui s'intéresse à étudier les interactions énonciatives des individus. De tels termes et expressions ne manquent pas du tout de la rhétorique et des figures de style. On y trouve évidemment la personnification des choses, la métaphore, la métonymie et au-dessus de tout la comparaison. Ce sujet pourrait subir une étude élargie d'un 
point de vue à la fois lexicologique, étymologique et sémantique.

\section{Bibliographie sélective}

- Alrabaa S., «The use of adress pronouns by Egyptian adults〉 In Langue Française 35: 117-119, 1977.

- Baylon C., Mignot X, La Communication, Natthan, 1991.

- Carade, F., Dictionnaire du francais argotique et populaire, Larousse, 1998.

- Dubois. J., Dictionnaire de linguistique et des sciences du langage, Larousse, 1994.

- Eduard, R., Dictionnaire des injures, Paris, Tchou, 2004.

- Goffman E., Les rites d'interaction, Minuit, 1974.
- Guiraud, P., L'argot, Paris: PUF, 1969.

- Huston N., Dire et interdire: éléments de jurologie, Paris, Payot, 1980.

- Lemonier, M., Insultes, gros mots et injures, petit dictionnaire des insultes de A à Z, Mini poche. City Editions, Hachette, 2009.

- Parkinson, D. B., Constructing the Social context of communication. Terms of Address Egyptian Arabic, Mouton de Gruyter, 1985.

- Pergnier, M., Le Mot, Paris, PUF, 1986.

- Rouayrenc, C., Les Gros mots, Paris, PUF, 1998.

- Vion, R., La communication verbale. Analyse des Interactions, Hachette, 1992. 